\title{
The time for radionuclide ventriculography resurrection is coming
}

\author{
Raffaele Giubbini, $M D,{ }^{a}$ and Elisa Milan, $M D^{b}$ \\ a University of Brescia, Brescia, Italy \\ b San Giacomo Apostolo Hospital, Castelfranco Veneto, TV, Italy
}

Received Jul 5, 2015; accepted Jul 10, 2015

doi: $10.1007 / \mathrm{s} 12350-015-0245-\mathrm{x}$

\section{See related article, pp. 1128-1138}

Left ventricular ejection fraction (LVEF) is the most widely used and easily available index of LV function. Almost all patients with suspected or documented heart disease undergo LVEF assessment at some point. There are nowadays, many different, invasive and non-invasive techniques for measuring LVEF including contrast ventriculography, echocardiography, magnetic resonance imaging (MR), computed tomography angiography (CT), and nuclear techniques. The nuclear techniques include the equilibrium radionuclide angiography (ERNA) referred to also as multigated angiography (MUGA), first-pass radionuclide ventriculography, gated single-photon computed tomography (SPECT) with myocardial perfusion imaging, and SPECT ERNA.

Until the 1970s, the most commonly employed method of determining LV volumes and EF was LV contrast angiography performed at the time of cardiac catheterization. In 1971, Strauss et al ${ }^{1}$ described a new revolutionary "scintiphotographic method for measuring LVEF in man without cardiac catheterization." As reported by Ashburn et al in the Seminars in Nuclear Medicine in $1973,{ }^{2}$ this procedure made it possible to have multiple determinations of LVEF and volumes without additional administrations of radioactivity, allowing acquisition in different projections to evaluate wall motion of the various surfaces of the heart. Our

\footnotetext{
Reprint requests: Raffaele Giubbini MD, University of Brescia, Piazza Spedali Civili, 1, Brescia, Italy; giubbini@med.unibs.it, raffaele.giubbini@unibs.it

J Nucl Cardiol 2016;23:1139-41.

$1071-3581 / \$ 34.00$

Copyright @ 2015 American Society of Nuclear Cardiology.
}

knowledge of the physiological changes of LV function during exercise was enhanced from the application of ERNA. The introduction of computers further improved the potential widespread use of this technique, ${ }^{3}$ preparing the grounds for semi-automated measurement of LV function. It is of historical interest to know that these results were obtained by acquiring data on $12 \mathrm{~K}, 16$ bits minicomputer system, recorded on magnetic tape and transferred them to a "large-scale digital computer as powerful as $200 \mathrm{k}, 32$ bits"!

In 1977, Borer et $\mathrm{al}^{4}$ demonstrated the feasibility of continuous monitoring and analysis of LV function during exercise allowing an accurate assessment of the presence and functional severity of ischemic heart disease. Global and regional dysfunction during exercise could easily be documented by ERNA. Specific features of ERNA were count- based measurement of LVEF independent from geometrical assumption and high reproducibility.

Van Royen et $\mathrm{al}^{5}$ compared side by side echocardiographic and quantitative radionuclide LVEF: their conclusion was that LVEF determined by ERNA and echocardiography showed good agreement. Both methods provided clinically valuable measurements of LV function. However, when a precision was required for reproducible measurements, ERNA was the method of choice.

Checking on PubMed with the following search terms, "radionuclide ventriculography," Radionuclide angiography," "ERNA," “MUGA," “scintigraphic angiocardiography," "radionuclide cineangiography," "scintiphotographic," " "gated scintiphotography," "radio isotopic angiocardiography," "radionuclide angiocardiography," or "ejection fraction," we found 2591 published papers on this topic. The use of ERNA reached its highest peak in the decade 1985-1995 (Figure 1), with a slow but progressive decline since.

The main reason for this decline is most likely the emergence of reliable competing modalities including 


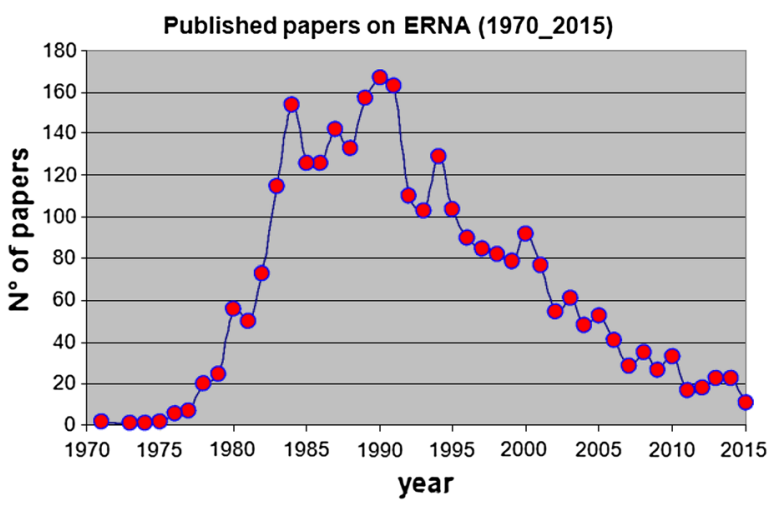

Figure 1. MedLine check of peer reviewed paper on equilibrium radionuclide angiography published in the last five decades.

MR, echocardiography, and gated SPECT with myocardial perfusion tracers. The present day application of ERNA is limited mainly to monitoring LV performance during chemotherapy or when the quality of images by other methods is suboptimal or could not be performed or when there is discordance between clinical judgement and imaging results. Many drugs, including anthracycline, alkylating agents, tyrosine kinase inhibitors, monoclonal antibodies, anti-metabolites, anti-microtubule agent, monoclonal antibodies, and drugs like interferon or bleomycin may affect LV function and require accurate LV function assessment during treatment.

The spectrum of cardiotoxicity may be associated with dysrhythmias, hypertension, pulmonary hypertension, right ventricular dysfunction, vasospasm, acute cardiac event, and heart failure (HF). Patients with a baseline LVEF $\geq 50 \%$ and a drop of $\mathrm{EF} \geq 10 \%$ during chemotherapy and a final EF below 50\% are at risk of HF, while patients with a baseline EF of $30-50 \%$ and a drop below $30 \%$ are at the highest risk of HF.

These patients should discontinue chemotherapy with doxorubicin and its analogs. For these reasons, a systematic follow-up is required with $\mathrm{EF}$ determination every $4-8$ weeks. ${ }^{6}$

Thus, considering the number of patients undergoing chemotherapy, how can we explain the decline in the use of ERNA in clinical practice when it has been clearly demonstrated to be useful for serial monitoring? ${ }^{7}$

There are several possible reasons to explain this circumstance: (1) planar imaging is limited by the overlap of different cardiac structures (right and left ventricle in the anterior projection, left ventricle and left atrium in the "best septal" view) affecting the calculation of LVEF, especially in the presence of mitral regurgitation, (2) The valvular function cannot be explored, (3) The volumes of heart chambers are only roughly determined, and (4) The radiation exposure and using the currently administered dose (740 MBq).
We should keep in mind that oncology patients are evaluated systematically by CT and PET/CT and/or other Nuclear Medicine procedures, and as recently pointed out by $\operatorname{Rehani}^{8}$; the individual patient dose received through diagnostic imaging may exceed an effective dose of $100 \mathrm{mSv}$. It is well known from the Hiroshima bomb observations that there is a linear relationship between exposure and incidence of cancer for an effective dose exceeding $50 \mathrm{mSv}$. The effective dose from $740 \mathrm{MBq}$ of $99 \mathrm{mTc}$ - albumin or $99 \mathrm{mTc}$ - labeled red blood cells is $5.18 \mathrm{mSv}$ according to ICRP publication $80 .{ }^{9}$ The question is: is it justified to administer $740 \mathrm{MBq}$ of radiolabeled red blood cells, with an additional $5.18 \mathrm{mSv}$ for each evaluation of LV function to such patients when other methods with no risk for exposure to ionizing radiations are currently available?

In this issue of the Journal of Nuclear Cardiology ${ }^{\circledR}$, Duvall et $\mathrm{al}^{10}$ showed that due to improved count sensitivity with the high-efficiency SPECT, a $50 \%$ reduction in injected activity is achievable while maintaining a short imaging times of 5 minutes, with further reduction possible at longer imaging times. It is therefore conceivable to hypothesize that an effective dose below $1 \mathrm{mSv}$ per study is achievable and hence less than 5-6 mSv for $\mathrm{LV}$ function monitoring during entire treatment course of cancer patients.

Gated blood pool SPECT angiography is a mature methodology and is well validated by a large body of literature as reported in the bibliography cited in the Duvall paper. The measurements of left and right ventricular EF are not affected by the overlap of different cardiac chambers and it can be corrected for attenuation and for scatter. All manufactures provide acquisition and processing protocols with resolution recovery for gated SPECT allowing the acquisition with half the administered activity for traditional dual headed SPECT.

The high quality of CZT SPECT can further improve the quality of gated blood pool SPECT. The accuracy of gated MPI-SPECT has been documented in comparison to $\mathrm{MR}^{11,12}$ but not yet for gated blood pool SPECT by CZT cameras. A comparison between MR and CZT gated blood pool ventriculography for measuring right and left ventricular EF and volumes will be an important next step.

\section{Disclosure} disclose.

The authors have no conflict of interest nothing to

\section{References}

1. Strauss HW, Zaret BE, Hurley PJ, Nataranjan TK, Pitt B. A scintiphotographic method for measuring left ventricular ejection 
fraction in man without cardiac catheterization. Am J Cardiol 1971;28:575.

2. Ashburn WL, Kostuk WJ, Karliner JS, Peterson KL, Sobei BE. Left ventricular volume and ejection fraction determination by radionuclide angiography. Semin Nucl Med 1973;3:165-76.

3. Green MV, Ostrow HG, Douglas MA, Myers RW, Scott RN, Bailey JJ, Johnston GS. High temporal resolution ECG-gated scintigraphic angiocardiography. J Nucl Med 1975;16:95-8.

4. Borer JS, Bacharach SL, Green MV, Kent KU, Epstein SE, Johnston GS. Real-time radionuclide cineangiography in the noninvasive evaluation of global and regional left ventricular function at rest and during exercise in patients with coronary artery disease. N Engl J Med 1977;296:839.

5. van Royen N, Jaffe CC, Krumholz HM, Johnson KM, Lynch PJ, Natale D, et al. Comparison and reproducibility of visual echocardiographic and quantitative radionuclide left ventricular ejection fractions. Am J Cardiol 1996;15(77):843-50.

6. Schwartz RG, Jain D, Storozynsky E. Traditional and novel methods to assess and prevent chemotherapy-related cardiac dysfunction noninvasively. J Nucl Cardiol 2013;20:443-64.

7. Mitani I, Jain D, Joska TM, Burtness B, Zaret BL. Doxorubicin cardiotoxicity:prevention of congestive heart failure with serial cardiac function monitoring with equilibrium radionuclide angiocardiography in the current era. J Nucl Cardiol 2003;10:132-9.

8. Rehani MM. Challenges in radiation protection of patients for the 21st century. Am J Roentgenol 2013;200:762-4.

9. ICRP. Radiation dose to patients from radiopharmaceuticals: Addendum 3 to ICRP Publication 53. Ann ICRP 2008;38(1-2): 1-197.

10. Duvall WL, Guma-Demers KA, George T, Henzlova MJ. Radiation reduction and faster acquisition times with SPECT gated blood pool scans using a high efficiency cardiac SPECT camera. J Nucl Cardiol 2015. doi:10.1007/s12350-015-0214-4.

11. Giorgetti A, Masci PG, Marras G, Rustamova YK, Gimelli A, Genovesi D, Lombardi M, Marzullo P. Gated SPECT evaluation of left ventricular function using a CZT camera and a fast lowdose clinical protocol: Comparison to cardiac magnetic resonance imaging. Eur J Nucl Med Mol Imaging 2013;40:1869.

12. Cochet H, Bullier E, Gerbaud E, Durieux M, Godbert Y, Lederlin M, Coste P, Barat JL, Laurent F, Montaudon M. Absolute quantification of left ventricular global and regional function at nuclear MPI using ultrafast CZT SPECT: Initial validation versus cardiac MR. J Nucl Med 2013;54:556-63. 